\title{
IMAGINATION, RELIGION, LANGUE \\ Trois vecteurs de la révolution pédagogique fichtéenne des Discours à la Nation Allemande*
}

\author{
Manuel Tangorra** \\ http://orcid.org/0000-0002-8989-4199 \\ manuel.tangorra@uclouvain.be
}

\begin{abstract}
RESUMÉ Si l'éducation a pu trouver sa place dans le noyau anthropologique des Lumières philosophiques, c'est dans la mesure où elle permettait l'intellection d'un horizon universel pour le genre humain. Fortement influencé par cette matrice conceptuelle dans sa jeunesse, Fichte va être cependant le témoin des limites de ce paradigme pédagogique à l'heure de saisir les processus historiques d'apprentissage collectif. L'hypothèse qui anime cet article identifie dans Les Discours à la Nation Allemande une réflexion pédagogique qui fait de l'éducation le travail d'activation des potentialités créatives d'une société pour construire, depuis sa situation affective et existentielle, sa propre destinée commune. Dans un contre-point avec la conceptualisation kantienne et à travers trois dimensions fondamentales de la révolution pédagogique des Discours (Imagination, Religion et Langue), nous aspirons à montrer comment Fichte dépasse le plan des conditions formelles de l'éducabilité et propose un savoir sur la genèse effective de l'apprentissage dans l'immanence de l'autodétermination des sujets historiques.
\end{abstract}

Mots-clefs Fichte, education, imagination, religion, langue.

ABSTRACT By allowing the intellection of a universal horizon for the human kind, education has found its place in the anthropological core of Enlightenment. Strongly influenced by this conceptual frame in his youth, Fichte

* Article submitted on 25/10/2019. Accepted on 16/05/2020.

** Université Catholique de Louvain. Louvain-la-Neuve, Belgique.

KRITERION, Belo Horizonte, nº 148, Abr./2021, p. 211-233 
has however witnessed the limits of this pedagogical paradigm when it comes to understanding the historical processes of collective learning. The hypothesis followed in this article recognizes, in the Addresses to the German Nation, a pedagogical reflection that conceives education as the work of activating a society's creative potential, of awaking a capacity to build, from an affective and existential situation, its own common destiny. In a counterpoint to Kantian conceptualization and through three fundamental dimensions of the pedagogical revolution of the Addresses (Imagination, Religion and Language) we aim to show how Fichte goes beyond the formal conditions of educability and proposes a knowledge on the effective genesis of learning in the immanence of the selfdetermination of historical subjects.

Keywords Fichte, education, imagination, religion, language.

\section{Introduction}

L'un des traits caractéristiques parcourant les expressions aussi bien philosophiques que scientifiques de la pensée des Lumières est sans doute le développement d'un paradigme pédagogique comme matrice conceptuelle pour la compréhension de l'existence humaine. En ce sens, "l'éducation du genre humain » constitue - au-delà du titre d'un ouvrage capital - l'insigne par le biais de laquelle l'Aufklärung cherche à se définir elle-même comme projet culturel, politique, religieux. Le travail d'autoformation du sujet rationnel nécessite, d'une part, une considération des institutions concrètes pour son développement : une réflexion sur la famille, l'école, l'université. Mais de l'autre, et de façon plus fondamentale, ce paradigme pédagogique doit être capable de projeter un horizon universel pour l'humanité. L'éducation participe dès lors du noyau anthropologique de la modernité qui permet de penser une destinée commune pour l'espèce, l'activité éducative rendant possible la reconnaissance des existences empiriques différentes dans un seul et même cheminement spirituel.

D'un certain point de vue, la philosophie de Fichte est l'un des prolongements les plus retentissants de ce mouvement qui érige l'éducation comme condition de possibilité d'une sociabilité rationnelle universelle. Dans le sillage kantien, Fichte conçoit, dès ses premiers écrits, le travail philosophique lui-même sous une perspective pédagogique qui fait du savant non plus un amasseur d'érudition métaphysique, mais un agent qui convoque les sujets à la critique de l'usage de leur raison et à l'apprentissage réciproque dans l'exercice 
de leur liberté1. Cependant, au cours des années, la pensée de Fichte chemine de plus en vers une philosophie qui ne se borne plus à la connaissance génétique des structures transcendantales du sujet et des principes éthico-politiques impliqués, mais qui se focalise dans la genèse effective de cet ordre normatif au sein des subjectivités concrètes ${ }^{2}$. Le présent travail sera voué aux implications pédagogiques de ce développement de la pensée fichtéenne, par le biais duquel notre auteur entame une nouvelle réflexion sur l'apprentissage, d'emblée située dans la concrétude culturelle, affective et langagière des libertés effectivement existantes.

À nos yeux, les Discours à la Nation Allemande achèvent ce dépassement fichtéen de la rationalité pédagogique de l'Aufklärung, qui germait depuis les textes de jeunesse, mais qui se manifeste désormais dans une théorie de l'éducation comme processus d'activation de la créativité individuelle et collective des sujets historiques. Certes, l'inscription d'une réflexion philosophique dans la matérialité d'une communauté nationale a été interprétée par de nombreux lecteurs, certains d'entre eux dans une apologie partisane (Cf. Treitschke, 1907, pp. 241-247 et Bergmann, 1933), comme le signe d'une conversion romantique de notre auteur, en rupture avec sa jeunesse

1 II faut cependant noter que, déjà depuis ses premiers écrits, Fichte s'intéresse aux conditions matérielles d'un progrès vers l'autonomie rationnelle. Dans les Conférences sur la destination du savant par exemple, la tâche pédagogique du philosophe consiste à indiquer le telos de forme transcendantale de l'autonomie, mais celle-ci se complète par un travail éducatif qui doit saisir la situation historico-culturelle, pour connaitre les conditions qui permettent, à chaque époque historique, de progresser vers une telle finalité. (Fichte, 1962ff [dorénavant GA] I, 3, pp. 56-57) Dans ce sens, et en reprenant la lecture de Jean Christophe Goddard, nous croyons que c'est l'approfondissement de la même intuition de jeunesse, et non pas une rupture, ce qui mène à Fichte à considérer l'agir fini, situé et affectif du sujet, non plus simplement comme condition d'effectuation, mais comme le principe herméneutique même de la téléologie absolue de la liberté (Cf. Goddard, 1999, p. 36).

2 Deux textes fondamentaux, antérieurs aux Discours, expriment déjà très bien cette inquiétude, en mobilisant notamment la forme du discours religieux comme perspective capable de saisir le fond subjectif des convictions à l'œuvre dans l'engagement historique de la liberté. D'un côté, il s'agit du Caractère de l'époque actuelle, présenté en cours en 1804-1805, lequel propose une analyse du cadre situationnel de l'appel absolu à la réalisation de la liberté. Dans la $16^{\circ}$ ème leçon Fichte soulève les limites de la « moralité pure » comme horizon pour la détermination de l'agir libre des humains, et fait recours à la religion comme pratique spirituelle capable d'engager l'intériorité affective du sujet dans une œuvre collective. A cette occasion, dans le but d'interroger la genèse de l'adhésion à la norme morale dans la personne, Fichte se demande « ce que l'homme moral nommait 'devoir' et 'commandement', qu'est-ce que pour lui ? » (GA, I, 8, p. 381). Dans le même sens et peu de temps après, l'Initiation à la vie bienheureuse propose la " moralité supérieure » en tant que " point de vue » spirituel permettant de saisir l'enracinement existentiel de la genèse des horizons normatifs de l'action: "La loi du troisième point de vuen'est pas uniquement, comme celle du deuxième, une loi qui ordonne ce qui est présent, mais plutôt une loi qui crée, dans ce qui est présent [innerhalb des vorhandenen], le nouveau et l'absolument non présent. » (GA, I, 7, p. 109). Mais ce ne sont pas les textes dits " populaires » les seuls qui pointent vers un savoir de l'autogenèse de la vie dans les différents points de vue qui projettent des images de celle-là. Dans la Doctrine de la science de 1804, Fichte expose la fonction systématique de cette perspective, lorsqu'il annonce la nécessité de comprendre la reconstruction " personnelle et authentique ", et donc particulière, de cette activité absolue d'automanifestation du savoir : " Le savoir absolu est institué objectivement et dans son contenu, et si c'était là notre seul but, alors notre travail serait achevé. Mais la question se pose encore de savoir comment nous qui sommes devenus ce savoir, nous le sommes devenu ; et pour le cas où cela serait soumis à d'autres conditions, quelles sont-elles ? » (GA, II, 8, p. 379). 
jugée rationaliste et républicaine (Cf. Andler, 1917, préf; Vermeil, 1939, p. 1017; Popper, 1945, pp. 53-55; Talmon, 1963, p. 174; Dumont, 1992, pp. 122-131; Berlin, 1992, p. 248; Losurdo, 1994, p. 172). Par fortune, depuis un temps déjà considérable, la Forschung fichtéenne ${ }^{3}$ nous épargne de devoir revenir sur l'incompatibilité du concept de « peuple originaire » (Urvolk) des Discours avec un quelconque nationalisme suprématiste. Nous le verrons, c'est justement la compréhension de la nation en tant que société d'apprentissage qui permet à Fichte de prendre distance par rapport à l'essentialisme d'inspiration romantique, en faisant de la communauté nationale l'espace d'une activité éducative, ancrée dans l'histoire, mais radicalement ouverte à tout sujet désirant participer à ce perfectionnement réciproque.

Afin d'examiner l'hypothèse avancée, à savoir celle d'une révolution pédagogique à l'œuvre dans l'exhortation éducative des Discours, nous aurons affaire à trois dimensions fondamentales de celle-ci. Dans un premier temps, il s'agira d'analyser la fonction de l'éducation de l'imagination, c'est-à-dire, la formation de la faculté de créer des images, dont le rôle est fondamental pour la production culturelle d'un horizon éthique partagé. Puis, il sera question de déceler le déplacement radical de l'enseignement religieux, compris dorénavant comme praxis de désaliénation spirituelle. Finalement, nous nous consacrerons aux enjeux de la pédagogie de la langue proposée par Fichte, laquelle tire au clair la nécessité de penser l'enracinement matériel et affectif de la liberté dans son expression historique.

\section{1. Éduquer l'imagination}

En première approche, les Discours constituent une intervention de conjoncture face à l'occupation napoléonienne du territoire allemand, qui se consolide avec la bataille de Jena vers la fin de 1806. Mais le texte s'adresse, avant tout, à un contexte spirituel où le souci fondamental est la colonisation culturelle et symbolique à l'égard de laquelle l'incursion militaire n'en est que le signe extérieur. Sous cet aspect, l'entreprise napoléonienne est la manifestation d'une nette déroute despotique des idéaux des Lumières et des nous pouvons repérer la progressive récupération des Discours chez Victor Basch (1914, pp. 711-793), Martial Gueroult (1974, pp. 233-246), Alexis Philonenko (1984. pp. 175-211) et Alain Renaut (1988, pp. 189-205). En langue allemande, nous sommes obligés de mentionner le travail de Reinhard Lauth (1992, pp. 197-230) et d'Erich Fuchs (2010, pp. 267-284.) Finalement, cet article s'inspire en grande mesure des recherches menées par Jean-Christophe Goddard (1994, pp. 63-177; 2012.) et Marc Maesschalck (1994, pp. 245-255; 1996; 2000). 
principes juridico-politiques de l'État rationnel qui se sont scindés de leur genèse dans la vie concrète des peuples. L'égoïsme le plus abject, hypostasié en emprise impériale, est parvenu à s'approprier des leitmotivs de la Révolution, des avancés de la pensée moderne, ou encore, au dire du dernier Fichte, de la « force formelle de la volonté morale » (GA, II, 16, p. 59). En ce sens, « l'ancienne éducation », qui s'oriente selon un idéal préétablit de pureté morale, est amenée à collaborer avec l'expansion du régime de la propriété qui érige son intérêt en loi universelle et conçoit le reste de l'humanité comme une " masse inerte et aveugle » (Idem, Ibidem) qui doit être guidée, moyennant une coaction externe, par le chemin univoque du progrès.

Dans un mouvement de contre-formation face à ce dispositif d'aliénation culturelle, le projet d'éducation national de Fichte tente de performer un espace pour l'autodétermination d'une communauté historique qui se décide à tracer son propre horizon civilisationnel. À cet égard, la contextualisation de l'apprentissage ne suffit pas, la construction collective d'une destinée sociale nécessite une nouvelle capacité pour créer des images de la réalisation mondaine de la liberté. Pour cette raison, la nouvelle éducation est, fondamentalement, l'incitation à l'exercice de l'imagination productive. Il ne s'agit pas ici de la bien connue imagination reproductrice, celle qui remet et associe les éléments donnés au préalable dans l'intuition, mais d'une créativité radicale, d'une expression sensible émergée de l'activité spirituelle. Une telle dimension productrice se trouve bien évidement déjà chez Kant, pour qui l'imagination construit « spontanément » les schémas qui sont la condition transcendantale de l'objet de connaissance (Kant, 1968, p. 148 [KrV b151-152]). Cependant, comme le remarquait déjà Heinz Heimsoeth ${ }^{4}$, du moment où chez Kant cette productivité trouve sa fonction primordiale en tant que condition transcendantale de la raison théorique, elle est orientée par l'entendement dans le but de synthétiser, a priori, la multiplicité sensible. L'imagination fichtéenne - dans ses premières formulations (Cf. GA, I, 2, p. 361) aussi bien que dans sa déclinaison plus tardive dans une théorie de l'image (Cf. GA, II, 9, p. 308) - radicalise le mouvement créateur en situant l'imagination en tant que faculté d'autoconstitution pratique

4 « Le concept kantien d'imagination productive - d'où émerge la pensée de Fichte - s'était limité à permettre l'exercice de la légalité spontanée de l'entendement dans le sensible et le multiple. [...] Telle est en revanche l'idée centrale dans la conception fichtéenne de la vie spirituelle, d'après laquelle l'intuition spirituelle ne peux pas être l'appréhension d'un objet donné en tant qu'être - comme chez Platon, Descartes ou, dans une certaine mesure, chez Kant - mais elle doit être une intuition créatrice, une force de préfiguration [Vorbildungkraft] [...] »(Heimsoeth, 1923, p. 103). 
de la liberté, et donc comme capacité infinie de construction d'un modèle de soi et d'un idéal du monde, capables d'engager l'action du sujet ${ }^{5}$.

La nouvelle éducation prend ainsi ses distances de l'idée de l'instruction comme simple transmission de connaissances - bien que le partage de savoirs trouve une place au sein de la nouvelle institution scolaire - mais aussi de la pratique pédagogique conçue comme induction de préceptes universels pour la conduite. L'enseignement pratico-morale est certes l'objectif fondamental, sauf que celui-là ne se comprend plus comme réforme du caractère vis-à-vis de la purification du mobile de l'agir, mais comme initiation à la production d'images qui puissent préfigurer la destination de l'humain à la liberté dans l'action effective. C'est ainsi que Fichte dépeint une activité qui ne se borne pas au respect de la forme rationnelle de la volonté, mais qui trace positivement un « modèle » ou une " préfiguration » [Vorbild] concrète, une réalisation de la liberté absolue dans la vie présente du sujet individuel et de la nation:

Cette faculté de forger spontanément [selbstthätig] des images qui ne soient aucunement de pures reproductions [Nachbild] de la réalité, mais qui soient capables de devenir des préfigurations [Vorbild] de celle-ci, constituerait le premier élément dont devrait partir la formation de l'espèce humaine par l'éducation nouvelle. Les forger spontanément, ai-je dit, et donc de manière que l'élève se les crée par sa propre force, et nullement en devenant simplement capable d'appréhender passivement l'image qui lui est indiquée par l'éducation, et de la comprendre suffisamment pour la reproduire telle qu'elle lui a été donnée comme s'il s'agissait seulement de se la rendre disponible [Vorhandensein]. La raison qui conduit à exiger la vraie spontanéité dans cette production d'images est la suivante : c'est seulement à cette condition que l'image ainsi forgée peut attirer à soi le plaisir effectif de l'élève. (GA. I, 10, p.120)

Si l'imposition des images-copies [Nachbild] peut conduire uniquement à l'immobilisme et à la résignation face à l'existent, la pédagogie du modèle permet de déclencher une transformation des structures sociales bloquant la construction d'une communauté rationnelle des libertés. Ainsi la formation morale de l'humain ne vise plus uniquement l'autolimitation sacrificielle des mobiles sensibles vis-à-vis les dictats transcendantaux de la raison pratique.

5 Dans la Critique du Jugement Kant explore certes des fonctions de l'imagination où celle-ci n'est pas déterminée par un concept de l'entendement. Cependant, même dans des tels cas - où l'imagination appréhende une forme de finalité sans la détermination conceptuelle d'une fin (Cf. Kant, 1902ff. [Dorénavant AA], IX, p. 489) - le matériel sensible de cette configuration schématique ou symbolique est toujours supposé comme une donnée externe simplement reçue. Avec le déplacement de l'imagination en tant qu'acte pratique d'autodétermination, Fichte questionne également l'extériorité représentative de la matérialité empirique. Ainsi, sur le plan pragmatique de la pédagogie, l'éducation doit stimuler une activité imaginative qui incorpore, en tant que dimension immanente de son effectivité, l'élément sensible au sein duquel elle se déploie. L'attention portée à la matérialité sémiotique de la langue au sein d'une théorie de la créativité sociale - analysée dans notre dernier alinéa - se suit à notre avis de cette transformation conceptuelle. 
Le travail éducatif invite désormais à s'accoupler à la liberté originaire de l'esprit par le biais d'une image qui se déploie dans l'immanence de l'acte d'autodétermination. Fichte souligne emphatiquement la nécessité d'envisager une composante affective positive lors de cette création imaginative (Idem, p. 120). Il y a un plaisir dans le mouvement de se faire image de la vie originaire, plaisir qui diffère, bien entendu, du plaisir individuel abandonné à la jouissance du donné : il s'agit d'un plaisir actif(Idem, Ibidem) que l'éducation doit étayer et approfondir afin de garantir un engagement affectif avec la construction communautaire d'un nouvel ordre éthique.

L'éducation de l'imagination est d'emblée orientée à l'agir, elle consiste elle-même en une praxis réflexive de libération symbolique. En conséquence, les intellectuels tenus pour catalyseurs d'un tel mouvement ${ }^{6}$ doivent porter leur attention à la dimension opérative des figurations morales, politiques, religieuses ; à l'aspect procédural de la production des horizons de sens, ceci afin d'empêcher la répétition mécanique reproduisant la tutelle extérieure. Mais, comment assurer que l'image projetée constitue un modèle autoactivement engendré et non pas imposé ? Comment être certain que l'élève tâchera, « avec amour ardent » (Idem, p. 128), de réaliser ce nouvel ordre une fois exempté du cadre scolaire ? À cet égard, peu importe si les idées inculquées sont traditionnelles ou révolutionnaires, rien dans leur contenu ne peut donner des garanties. Si la genèse d'un tel ordre symbolique ne prend pas ses racines dans la liberté de l'éduqué, les concepts transmis seront l'opérateur idéologique d'une relation hétéronome avec la réalité. Seul l'agir par le biais duquel l'élève - et tout sujet historique au sein d'une communauté de perfectionnement mutuel - s'apprête avec plaisir à réaliser l'ordre imaginé dans sa situation mondaine, nous fournit un indice d'un modèle qui émerge depuis sa liberté la plus intime. L'institution éducative doit donc viser un engagement pratique des sujets avec la totalité sociale, une action positive qui dépasse la seule observance du système formel des peines et châtiments, lequel possède certes une fonction, mais qui ne suffit pas pour fonder chez l'élève une adhésion véritable à la réalisation partagée de la liberté, au sein de laquelle il doit pouvoir se percevoir soi-même à titre d'acteur. Sur un autre niveau, la nation, en tant que grande

6 II faut avoir à l'esprit que le projet éducatif de Fichte soulève - particulièrement au long du dixième discours la nécessité d'une éducation intellectuelle, universitaire, pour certains individus de la communauté nationale. Cependant, la distinction d'une classe de savants ne doit pas cristalliser une asymétrie sociale, mais plutôt être le facteur dynamiseur de la mobilisation éducative, laquelle met en question, par principe, les stratifications sociales rigides. Nous suivons en cela Quentin Landenne : « Le seul pouvoir politique ou le seul privilège social du philosophe en tant que philosophe n'est donc en fin de compte que le pouvoir par définition provisoire de l'initiative pédagogique, de l'ouverture à une nouvelle dimension des relations sociales fondée sur une dynamique d'apprentissage collectif. » (Landenne, 2013, p. 253). 
communauté éducative, doit inciter de même à quelque chose de plus qu'au simple respect des cadres juridiques. Seule la praxis active, la participation volontaire dans une œuvre commune qui transforme l'état de choses, est la preuve d'un modèle qui s'enracine dans le fond affectif de la personne, et ainsi, dans la vie du peuple, conçue en tant que puissance d'imaginer de manière autonome la transformation de ce qui existe.

Permettons-nous, afin de mesurer la portée révolutionnaire de cette formation à la préfiguration, de revisiter brièvement les postulats pédagogiques de Kant, qui représentent sans doute un point d'aboutissement des réflexions de l'Aufklärung sur l'éducabilité de l'être humain. Au sein de son projet didactique, Kant fonde l'horizon téléologique de la formation spirituelle dans l'idéal moral, c'est-à-dire, dans la forme originale du devoir envers soi-même, qui doit réguler les efforts subjectifs et rendre intelligible le progrès culturel et politique. Voyons comment «l'idée de l'humanité » oriente la reforme morale et se révèle ainsi comme l'étalon fondamental pour la comparaison de tout agir humain, comme l'original universel appelé à être la mesure rationnelle de tous les sujets empiriques :

Beaucoup d'auteurs ont tout à fait négligé ou exposé de manière fausse la section de la morale qui contient la théorie des devoirs envers soi-même. Or comme on l'a dit, le devoir envers soi consiste pour l'homme à conserver la dignité de l'humanité en sa propre personne. L'homme s'adresse des reproches lorsqu'il a l'Idée de l'humanité devant les yeux. Cette idée est pour lui l'original [Original] auquel il se compare. (Kant, AA, IX, p. 489)

L'agir individuel et collectif empirique se rapporte, moyennant les cadres socio-culturels de formation, à une finalité formelle qui s'érige comme point de repère universel permettant d'évaluer les efforts des individus pour se libérer de leur détermination sensible. Certes, cette finalité ne désigne pas despotiquement un état de chose à atteindre, elle évoque la loi de la propre autonomie et engage une normativité catégorique en raison, justement, de son indépendance vis-à-vis de tout conditionnement externe à la liberté du sujet. Cependant, du moment où le telos qui sous-tend le parcours formatif demeure formel, le rapport que les sujets historiques entretiennent avec la destination universelle de l'humanité se définit fondamentalement de manière restrictive ${ }^{7}$. Ainsi, à l'heure de la moralisation de l'existence empirique, l'éducation ne

7 Gérard Lebrun expose ce concept kantien avec toute clarté: « L'important c'est qu'il n'y ait pas plus de genèse historique de la moralité que de pédagogie morale ou de maturation d'un peuple pour la liberté. [...] En fait, la prémonition de la moralité dans la culture n'est jamais que négative ». (Lebrun, 1970, pp. 480-481) 
peut que s'appuyer sur une discipline négative, notamment sur l'inhibition des passions dans le mobile de l'intention (Idem, pp. 486-487).

Le Vorbild fichtéen permet de penser autrement la relation éducative entre le sujet empirique et l'universalité morale. Il ne s'agit pas de se contenter de l'intellection d'un système transcendantal de principes, auquel il faudrait comparer, après coup, les efforts concrets des individus. Bien autrement, il est question d'activer une relation créative avec la vie suprasensible, relation qui s'affirme pleinement dans le présent situationnel des sujets. Le modèle n'est pas le symbole ${ }^{8}$ d'une perfection morale qui s'échappe dans un au-delà insaisissable, il est, en revanche, l'imagination en contexte d'un mode de réalisation de l'universalité morale. C'est un acte pré-figuratif qui rend visible l'invisible, (GA, I, 10 p. 196), non par une concordance de l'action sensible avec un schéma rationnel a priori - dynamique éducative qui habilite toujours une hiérarchisation des existences selon un paradigme unique de civilisation -, mais par l'expression, dans sa finitude comme modèle historico-culturel de quelque chose qui excède son être phénoménique, un surplus [Mehr] (Idem, p. 193) qui émerge de toute action subjective se reconnaissant dans la genèse de la liberté universelle de la vie 9 .

Le clivage original-copie, fondement de pratiques éducatives aliénantes au cours de l'histoire moderne, se trouve explicitement abrogé dans la proposition pédagogique des Discours. L'universalité du genre humain ne se noue plus à un idéal original qui survole les individus et les sociétés, mais elle dénote, avant tout, l'infinitude originaire de la liberté qui se rend consciente dans chaque création historique, comme l'unité illimitée qui dans chaque manifestation se détache de sa limitation, en s'ouvrant à une déterminabilité infinie qui augure la continuité du travail éducatif. La destination s'actualise dans l'opération effective de l'imagination, non pas comme tentative de représentation de sa

8 C'est justement à partir du symbolique que Kant réfléchit à un usage non schématique de l'imagination et à la possibilité donc d'une expression sensible des idées morales [Versinnlichung sittlicher ldeen] (Cf. Kant, Critique de la faculté de juger, AA, V, p.356). Cependant, le symbole demeure fondé sur une procédure analogique, et bien qu'il ne s'agisse plus de l'analogie classique entre des objets du même genre (d'ailleurs impossible en s'agissant de l'idée de liberté ou de Dieu) elle repose sur un type spécial de similitude, à savoir entre la forme réflexive du jugement esthétique et la réflexion morale. Le modèle de Fichte, diffère de l'analogie symbolique, car il n'est point l'indice esthétique d'un concept universel, mais l'expérience pratique de la vie universelle dans une mise en image nécessairement située.

9 Dans cette conception du "surplus" de l'activité créative par rapport à son produit empirique, se rend effectif un nouveau rapport à l'absolu, distinct du sublime kantien. D'après les mots de Jean Christophe Goddard: " La contradiction du fini et l'infini est interne au tracé imageant de l'imagination, qui ne cherche nullement à figurer l'irreprésentable, mais engendre toujours l'infini sur le bord de son tracé, comme ce qui se détache de la limitation (Goddard, 1994, p. 169). 
forme, mais comme incarnation du principe créatif de l'esprit humain ${ }^{10}$. Le modèle est une mise en image de la liberté, en tant que répétition de son être commencement absolu, c'est-à-dire, en tant que réactivation de celle-ci dans sa puissance originaire. La conscience singulière, dans la reconstruction imaginative d'une image de soi et d'un monde moral, ne s'adresse pas à sa liberté par une formalisation de son devoir-être, mais par un mouvement de vivification de cet impératif, qui s'inscrit dans l'histoire comme principe créateur d'une destinée collective.

\section{2, Un nouvel apprentissage religieux}

Comme l'affirme Nick Cuneen, le travail imaginatif constitue la compétence opérative fondamentale pour toutes les dimensions ultérieures du projet éducatif des Discours, du moment qu'il permet la « déduction » autonome d'un contenu éthique et « l'association d'un plaisir à ce contenu » (Cuneen, 2016, p. 158). Cela explique pourquoi Fichte trouve dans le discours religieux une déclinaison exemplaire de ce rapport entre modèle pratique de l'action et universalité morale. Si l'éducation religieuse peut être considérée en tant que « visée ultime » (GA, I, 10, p. 193) du nouveau projet éducatif, ce n'est point par une prééminence a priori du domaine religieux, mais par une saisie de la « signification sociale de la croyance » (Maesschalck, 2000, p. 160), c'està-dire de l'effectivité de cette dernière dans la formation d'une subjectivité responsable vis-à-vis de son agir au sein d'une société de formation réciproque.

Certes, l'introduction de l'élève au religieux implique d'abord un décentrement des intérêts sensibles dans la mesure où elle vise à fonder les mobiles de l'action dans son appartenance à la vie supérieure de l'esprit (GA, I, 10, p. 132). Mais toute la singularité de la réflexion fichtéenne sur l'éducation religieuse se suit du fait de ne pas vouloir déduire une pédagogie religieuse ni des seuls impératifs de la raison pratique, ni d'une théologie spéculative. Pour penser l'initiation à la vie spirituelle il ne faut pas se borner à une méthodologie de la transmission doctrinale, mais réfléchir à un travail avec les conditions subjectives de l'accueil du suprasensible dans la source même des horizons pratiques de l'agir. Le troisième discours, voué spécifiquement à ce sujet, identifie la spécificité de la connaissance religieuse que l'élève doit acquérir pour consolider un engagement éthique. Il ne s'agit point d'une vérité

10 Sans doute cette doctrine pédagogique de la préfiguration se lie à ce que Christoph Asmuth définit comme le passage «de la représentation représentante à la représentation présentante » (von der repräsentierenden Vorstellung zu präsentierenden) caractéristique de la Bildlehre du Fichte tardif (Cf. Asmuth, 2010, p. 158). 
théologique imposée comme cadre prédonnée de l'action, mais d'un savoir réflexif à l'égard des convictions intérieures, à partir desquelles la volonté peut trouver librement sa détermination (Cf. Maesschalck, 1994, p. 253).

De même que pour l'ensemble de son projet pédagogique, Fichte est conscient du conflit que cette réactivation d'un pôle spirituel intérieur peut entretenir avec les discours institutionnalisés du religieux. Son diagnostic repère les dispositifs idéologiques qui réduisent la religion à une parole monolithique scindée des avatars de l'existence humaine, à une vérité à laquelle les sujets ne peuvent s'adresser que par une espérance abstraite ou par le mobile sensible de la peur, tous les deux déterminés par un prolongement de l'égoïsme mondain dans l'espoir du salut éternel (Cf. GA, I, 10, pp. 132133). La dévalorisation du temporel est issue d'une instrumentalisation de la croyance au service des rapports matériels de domination, qui usurpe le religieux pour détourner l'opprimé de son esclavage mondain, et « qui rejette vers le ciel ceux auxquels il ne veut accorder aucune place sur la terre » (Idem, p. 199). Dans ce cadre, les institutions religieuses sont devenues des agents d'une relation hétéronome entre une vérité absolue réifiée et la vie concrète de croyants, dans un rapport que Fichte n'hésite pas à qualifier de colonial. Les églises, anciennes responsables du travail éducatif, se sont érigées comme de véritables « colonies du ciel» [Pflanzstadt aus dem Himmel] (Idem, p. 240) ${ }^{11}$ dans la terre, comme des médiateurs d'une économie salvifique qui méprise les existences mondaines (Idem, p. 199) et qui les réduit à une épreuve vestibulaire de recrutement [anwerben] pour la communauté transcendante. Ce régime théologico-politique de colonisation spirituelle bloque tout rapport autochtone, créatif et innovateur au principe suprasensible qui pourrait s'entamer depuis l'interaction sociale des réalités terrestres.

Sur la base d'un tel bilan, l'éducation fichtéenne se propose comme une réappropriation libératrice d'un rapport intérieur et spontané - dans le sens d'autoactif [selbstthätig] - à l'absolu. Une telle spiritualisation du monde présent ne procède pas d'une nouvelle synthèse théorique, mais d'un travail avec le fond pulsionnel de l'action, là où se loge l'appréhension subjective du principe spirituel qui engendre, mais qui dépasse également, toute individualité finie. Les différents rapports de la conscience avec cette pulsion fondamentale déterminent l'existence mondaine du sujet et les horizons d'actions au sein

11 II n'y a pas de doute que ce terme fait référence, au moins dans le contexte des Discours, à une relation coloniale de subordination culturelle. L'autre apparition de ce mot dans le texte est liée à l'impérialisme culturel de l'Empire romain sur les peuples germains (G.A. I, 10, p. 206) lequel, il faut le dire, fonctionne dans le texte comme arrière-fond historique de la domination française napoléonienne. 
de celle-ci (Cf. Idem, pp.135-137). Ainsi, il est question d'enraciner toute représentation religieuse à ce lien intime et libre au suprasensible, qui ne s'offre pas dans la pleine autopossession consciente, mais dans l'illimitation ouverte par notre propre finitude qui projette une tâche pratique infinie, constituant celle-ci le véritable horizon de manifestation de l'absolu dans l'intériorité du soi. L'apprentissage religieux doit donc renvoyer toute figuration à sa genèse dans cet espace intérieur où la conscience se découvre dans son affectation fondamentale par un amour originaire ${ }^{12}$, à partir duquel elle peut se concevoir, cette conscience agissante dans le monde, comme manifestation de l'être absolu.

Pour ce faire, un basculement méthodologique s'avère nécessaire. Le nouvel enseignement religieux s'écarte de la simple transmission doctrinale et se focalise dans la stimulation d'une nouvelle compétence de représentation spirituelle, d'emblée orientée à la praxis dans le monde social. Suivant le mouvement analysé ci-dessus, il s'agit de mettre en question les images imposées de l'absolu par une réactivation d'un rapport créatif à celui-ci, à partir du contexte situationnel du sujet pratique. Cela implique avoir une considération spéciale envers la diversité des modèles religieux, dorénavant conçus comme des réceptions figuratives particulières - historiques et culturelles - de la vie universelle ${ }^{13}$. Ainsi, aucune parole institutionnalisée ne peut clôturer l'activité préfiguratrice de l'esprit, car celui-ci conteste constamment toute fixation représentative et appelle sans cesse à de nouvelles figurations (Idem, p. 137).

Une remarquable prise de distance à l'égard des réflexions kantiennes sur l'éducation, cette fois-ci dans le domaine religieux, se laisse ici entrevoir. $\mathrm{Au}$ moment de penser la formation religieuse, Kant s'appuie sur l'intellection d'une figuration originale rationnelle, à laquelle les modèles historiques de la religion révélée viennent s'ajouter pour étayer la croyance subjective. Ainsi, Kant distingue explicitement le noyau moral de la religion - la « religion universelle ${ }^{14}$ - qui doit s'enseigner suivant la méthode « socratique », c'est-àdire, où la raison autonome de l'élève est convoquée, des contenus de la religion révélée doivent se transmettre selon la procédure « cachectique-mécanique »

12 Notons sur ce point que le concept d'amour implique une double affectation, à la fois religieuse et éthique, du sujet éduqué. Comme l'affirme Luis Felipe Garcia : «Aussi bien dans sa dimension sensible que suprasensible, l'amour est cette sensibilité par laquelle l'enfant peut se détacher de son moi individuel au profit de la formation de l'idée d'un moi qui est, d'une part, irréductible à sa personne (dimension sensible de l'interpersonnalité) et, de l'autre, irréductible à la personne tout court (dimension suprasensible du prépersonnel) » (García, 2018, pp. 372-373).

13 Dans un rapport de la conscience croyante à l'universalité religieuse que Marc Maesschalck qualifie de « assomptif ». (Cf. Maesschalck, 2000, p. 69).

14 Cette distinction suit celle du traité sur la religion (Cf. Kant, AA, VI, p. 154). 
(Kant, AA, IX, p. 154). Ce dualisme méthodologique rend clair la réduction de l'historicité du religieux à une fonction instrumentale, la subordination des schématisations particulières de l'absolu - qui émergent dans des contextes sociaux, culturels et langagiers (comme on verra ci-dessous) - à une universalité rationnelle qui pourrait être enseignée dans sa pure structure rationnelle. La capacité figurative de la religion à transmettre dans l'apprentissage n'est pas créative mais expositoire, dans la mesure où les images de Dieu produites par une communauté de croyants sont des exemplifications d'un " archétype » moral a priori, qui se surajoutent pour renforcer la foi dans le pouvoir-être de cette perfection morale rationnelle.

Fichte en récuse explicitement cette dévalorisation de l'historique dans le cadre de l'éducation religieuse (GA, I, 10, pp. 140-141). Imposer mécaniquement le contenu imagé de la religion revient à négliger la nature pédagogique de la révélation et, en ce qui concerne le christianisme, l'enseignement libérateur de la personne historique de Jésus ${ }^{15}$. Si la religion est un vecteur central d'un projet pédagogique émancipateur, c'est dans la mesure où la croyance dans l'acte historique de la révélation habilite la compréhension de la vie éternelle comme tâche historique et sociale de libération collective. Le message éducatif de l'incarnation humaine de la divinité n'est pas le véhicule pour renforcer le respect aux commandements divins prédonnés, mais l'inauguration d'un nouveau rapport avec ceux-ci dans l'immanence des luttes humaines. Suivant ce chemin ouvert par le christianisme et rendu pleinement conscient par la Reforme, l'éducation religieuse doit habiliter une connaissance pratique du rapport à l'absolu, visant à la construction de figures positives d'action communautaire.

Par une telle reformulation de méthode, Fichte croit pouvoir resituer le religieux dans un processus intersubjectif de désaliénation des horizons spirituels. L'éducation a affaire au travail intime avec les convictions, à une nouvelle compétence réflexive à l'égard de notre pulsion fondamentale

15 La différence entre les christologies de Kant et Fichte ne fait pas l'objet ni de cet article ni de cet alinéa. II convient pourtant de signaler que leur distance dans la réflexion pédagogique se lie à une divergence dans la compréhension de la fonction éducative de la révélation chrétienne. Pour Kant, qui à ce propos emboite le pas à Lessing, la réalité historique de l'incarnation fonctionne comme exemplification (La religion dans les limites de la simple raison, AA, VI, p. 128) de l'idéal transcendantal du Christ, et donc sa tâche éducative se base dans une symbolisation de la religion universelle rationnelle. Fichte, tout particulièrement dans ces deniers écrits, s'écarte d'une telle réduction, en refusant d'abord l'hypostase métaphysique de la personne du Christ (Cf. L'initiation à la vie bienheureuse, G.A. I, 9, p. 188 et La doctrine de l'état [1813] G.A. II, 16, pp. 147-148.), mais surtout en comprenant la tâche pédagogique de Jésus depuis la facticité mondaine de son apparition (Cf. L'initiation à la vie bienheureuse, G.A. I, 9, p. 192). Ainsi, le message chrétien n'est pas l'exemplification d'une image originale de la bonne conduite présente de toute éternité dans la structure de la raison pratique, mais la manifestation et l'appel à une prise de conscience, subjective et personnelle, de la vie absolue comme liberté agissante dans l'histoire. 
permettant d'interpréter celle-ci comme manifestation historique et située de la vie et de s'orienter, par ce biais, dans la construction active d'une destinée collective. La connaissance promue n'est pas le savoir objectal d'un être éternel écarté des individus terrestres et leurs désirs ; elle implique au contraire une préfiguration de l'amour originaire dans les œuvres sociales de la liberté. L'engagement d'une communauté qui lutte pour son autodétermination pourrait ainsi trouver une nouvelle puissance symbolique dans un apprentissage religieux qui invite les sujets à faire de leur rapport à l'absolu la source d'une action qui n'attend pas le dernier jour pour saisir l'éternel, mais qui « sème » l'éternité dans la temporalité vivante et singulière d'un peuple historique (GA, I, 10, p. 199).

Une telle pédagogie du religieux se révèle particulièrement attentive à l'enracinement historique et affectif de la conscience croyante. Il s'agit de former une religiosité détachée de tout fétichisme fanatique de l'absolu, lequel s'origine justement dans l'exil du suprasensible au domaine ontologique de l'ultra-mondain. L'éducation doit montrer que la vie éternelle n'est pas ce qui commence au-delà du tombeau, mais qu'elle se trouve déjà là, « au cœur de cette époque » (Idem, p. 133), et dans l'appartenance à une communauté terrestre (Cf. Idem, p.202). Comme mouvement de libération spirituelle, l'apprentissage religieux procède d'une pédagogie de l'accueil de l'amour originaire dans le fond des convictions de l'agir matériel. L'éducation ouvre ainsi un nouvel espace de sensibilité, elle apprend à se laisser affecter - à se laisser saisir [ergreifen] (Idem, p. 194) - par la vie suprasensible dans le hic et nunc du croyant et dans son engagement avec les luttes de son temps présent.

\section{Langue originaire et socialisation de la parole}

Pour Fichte, l'élément linguistique joue un rôle vital au sein de cet effort pour penser une éducation attentive à la condition située du principe créatif de la liberté. L'apprentissage de la langue propre, l'exercice de créer des modèles esthétiques, politiques et religieux dans la langue dans laquelle se noue la vie matérielle est crucial pour un projet pédagogique basé sur la stimulation de la production individuelle et collective de l'ordre conceptuel. La langue est le symptôme de l'autoaffectation de la liberté à une réalité historico-culturelle, elle constitue donc le tissu sensible dans lequel s'enracine l'autonomie dans son expérience la plus radicale, dans l'acte de se donner un nom, de se dire. La forme morale de la destination, l'accord avec soi-même du sujet libre, se vivifie par une parole émergée de l'espace d'intelligibilité et d'affection collective constitué par la langue autochtone d'une communauté historique. 
La pédagogie qui vise l'acquisition d'une compétence exclusivement technique du langage ampute une dimension fondamentale de la créativité populaire. Avec toute l'estime porté à l'égard de Pestalozzi ${ }^{16}$ par Fichte - la grande référence de la pédagogie moderne en langue allemande -, ce dernier ne manque pas de signaler l'insuffisance d'une vision de l'apprentissage qui se limite à fournir l'élève d'un instrument de socialisation, d'un ensemble d'outils lui permettant l'accès à un champ social de reconnaissance déjà préétabli. Derrière une telle conception, se cache, d'après Fichte, un conformisme avec les structures présentes du social : la langue devient l'élément civilisateur qui habilite l'accès à une position sociale, mais un tel apprentissage mécanique naturalise l'ordre culturel auquel le sujet en formation devrait s'adapter ${ }^{17}$.

Cette critique de Fichte envers Pestalozzi est extensible, encore une fois, à la compréhension kantienne de l'enseignement langagier. Dans les passages d'ailleurs très succincts - de son traité sur l'éducation qui évoquent l'acquisition des facultés linguistiques, Kant se trouve dans la même lignée qui fait du langage une compétence technique, un instrument servant soit à la satisfaction des besoins physiques (AA, IX, p. 462), soit à l'exercice de l'abstraction et de la mémoire (Idem, p. 474). En définitive, ce qui prédomine chez Kant, et notamment dans ses réflexions anthropologiques, c'est une compréhension du langage en tant qu'ensemble de règles d'extériorisation de la pensée, les mots n'étant que des « désignations » (AA, VII, p. 192) voire des « notes » (AA, XXV, p. 770) de cette dernière. Dans ce cadre, les langues se voient caractérisées comme des « manières particulières de chaque peuple d'exprimer leurs concepts » (Idem, Ibidem), et donc hiérarchisées en tant que reflets des capacités intellectuelles des peuples. Ainsi, lorsqu'on enseigne une langue à travers ces règles constitutives - car d'après Kant « la grammaire devra venir en premier lieu dans l'étude des langues » (AA, IX, p. 475) - on a affaire à une méthode civilisatrice qui offre un nouveau moyen d'expression - peut-être en remplacement d'un autre plus défaillant ${ }^{18}$ - censé développer l'intelligence des sujets dans une énonciation transparente.

16 Dans les Dialogues Patriotiques, écrits moins d'un an avant les Discours, Fichte affirme que « la pensée de Pestalozzi est infiniment plus grande que Pestalozzi lui-même ». En ce qui concerne l'enseignement de la langue, l'erreur de Pestalozzi revient à ne pas développer jusqu'au bout ses propres prémisses : « Un usage de la langue comme celui qui recommande Pestalozzi, comme technique toujours déjà disponible pour ordonner les intuitions, c'est exactement ce qui conduit à l'omission de l'intuition et au verbalisme précoce, auquel en toute raison la propre théorie de Pestalozzi, là où elle se développe de manière conséquente, s'oppose avec tant de vigueur. » (Dialogues Patriotiques, G.A. II, 9, p. 442).

17 Pour la critique au caractère « assistentialiste » du projet d'alphabétisation de Pestalozzi, Cf. GA. I, 10, pp. 219-221.

18 « Par exemple, les péruviens manquaient au début d'un grand nombre d'expressions, mais, de suite, ils ont appris l'espagnol et on a découvert qu'ils devenaient progressivement plus intelligents ; on les appelait indiens 
En revanche, d'après Fichte, l'éducation linguistique se comprend comme une exploration des potentialités créatives de la parole. La langue n'est pas un instrument, un outil, un moyen dans le sens d'un ustensile contingent qui permet d'extérioriser des concepts qui le précèdent ou de viser des finalités qui le dépassent. Bien avant cela, la langue est la médiation originaire, non pas comme le nexus occasionnel entre des interlocuteurs préexistants, mais - pour prendre les mots des Dialogues Patriotiques - comme " médium » fondamental (GA, II, 9, p. 441). Les individus ne manipulent pas la langue comme un dispositif conventionnel, au contraire, celle-ci forme le milieu où prend racine la genèse même de la subjectivité, le bassin de gestation des identités personnelles et collectives. Comme le souligne Fichte lors de son quatrième Discours : « Les hommes sont bien plutôt formés par la langue que la langue ne l'est par les hommes » (GA I, 10, p. 145).

Comme milieu d'interaction spirituelle de la communauté, les facultés langagières ne se bornent donc pas aux signes empiriques donnés et l'éducation doit mobiliser une transgression permanente des significations figées. L'apprentissage de l'allemand, en l'occurrence, n'est donc pas l'introduction à un réservoir sémantique, mais l'initiation à une pratique d'autodépassement des formes idiomatiques existantes, par le biais d'une exploration constante des possibilités de la langue pour réussir une expressivité de la liberté dans la culture. Seul un fétichisme du langage pourrait abstraire une dimension de l'être empirique de la langue et la hisser au titre de fondement de la vie culturelle. Le nouvel apprentissage doit contrer une telle solidification et secouer les conditionnements sémiotiques de la création sociale, qui ne disparaissent jamais, mais qui doivent être subvertis en permanence par l'intuition spirituelle dans sa pulsion transformatrice. C'est sous cette optique que s'explique l'importance de la poésie au sein de l'éducation, non pas comme transmission d'un héritage patrimoniale, mais comme découverte des « expériences-limite » (Maesschalck, 2000, p. 155) du langage, qui ébranlent et élargissent l'être empirique de la langue pour la rendre l'élément d'expression du suprasensible (GA I, 10, p. 162).

Cetteidentification dela languecommesphèrepragmatiqued'autosubversion sémiotique, donne un sens spécifique au caractère ouvert de la communauté langagière des Discours. Il ne s'agit pas seulement de décréter résolument l'accueil à « quiconque qui veut poursuivre par la liberté le développement de la spiritualité » et de subordonner l'appartenance (même linguistique!) - « 
où qu'il soit né et quelle que soit sa langue »-à la communion dans un projet de réalisation de la liberté - « il fera cause commune avec nous » (Idem, pp. 195-196). Même avec cette bienveillance à l'égard de tout sujet désirant de participer, un schéma assimilationniste pourrait se mettre en place. Mais la radicalité de l'ouverture de la société d'apprentissage envisagée par Fichte va bien au-delà de cette politique de « portes ouvertes ». Elle se noue justement autour d'une compréhension de la langue comme milieu d'autotransformation identitaire. Dans le but poursuivi par l'éducation, la langue rejoint la pulsion pour manifester la vie spirituelle qui gît dans tout sujet libre; pulsion qui se manifeste dans un désir d'altération du monde présent et de soi-même dans son existence effective. Ainsi le désir de l'accord avec soi-même, de se rapporter à sa propre vérité suprasensible, se manifeste dans une affectation qui déclenche une diversification de soi radicale, cela se traduisant, sur le plan langagier, en une altération permanente des frontières linguistiques de l'expression. De cette manière, le travail pédagogique avec la langue ne favorise pas seulement une ouverture envers autrui, mais également une ouverture dans le propre rapport à soi-même, d'un sujet qui se reconnaît comme affecté par une non-identité constitutive au sein même de l'énonciation de sa liberté. Cette déclosion de soi retentit dans la matérialité sémiotique, laquelle - justement dans ses usages les plus spirituels et créateurs - est subvertie, diversifié et rendue réceptive d'autres univers de sens, par exemple dans l'adoption - « sans dommage pour leur liberté $»^{19}$ - de mots venant d'autres communautés langagières.

La langue allemande apparait ainsi comme l'occasion d'activer une praxis culturelle libératrice face à l'ancienne éducation qui promouvait, pour la formation de l'élite, l'acquisition des langues dites érudites qui distinguent un groupe d'éclairés, en charge de la production de l'ordre politique et culturel de la nation. La division sociale tranchante, entre les instruits et l'ensemble du peuple, manifeste la relation hétéronome d'une communauté d'interlocuteurs avec sa propre langue. Si Fichte identifie certaines langues comme « mortes », ce n'est pas à cause de leurs traits idiomatiques, mais à cause d'une scission avec leur propre genèse populaire (Idem, pp. 162-163), en raison d'un divorce avec la puissance originaire de l'expression, ce qui dérive dans une rigidification sémantique, qui peut certes jouir de perfection grammaticale (Idem, pp. 165-166), mais qui demeure isolée de la pratique communautaire du langage. La langue morte achève ainsi une aliénation de la parole, elle devient nécessairement étrangère [Ausland] - non par le fait de provenir d'ailleurs, 
d'avoir une origine différente -, mais par sa soustraction à la médiation créative de l'intersubjectivité ${ }^{20}$. La nouvelle éducation libératrice suppose ainsi une resocialisation de la parole, qui trouve dans la langue populaire la matière élémentaire pour la construction d'un nouvel ordre symbolique.

La supériorité que Fichte attribue à l'allemand par en dessus du français qu'incarne la domination culturelle liée à l'occupation politico-territorial - n'est donc pas à lire comme une suprématie patrimoniale de la langue germanique, mais à considérer d'après une analyse des conditions discursives et matérielles de production des horizons de signification. Le français est traversé par une coupure interne entre langue érudite et langue populaire qui le rend une langue étrangère en relation à sa propre création intersubjective et par conséquent étrangère dans l'instrumentalisation impérialiste envers d'autres peuples. Mais aucune langue - et donc même pas l'allemand - n'est a priori exemptée d'un processus d'aliénation opéré par des dynamiques élitistes de production symbolique. La quête éducative d'une langue originaire [Ursprache] - et non de la langue originale comme bien le précise Erik Fuchs (Fuchs, 2010, p. 274) -, d'une praxis originaire du langage, n'impose pas une langue déterminée comme substrat naturel exclusif d'une telle activité. Ce qui promeut Fichte, en revanche, c'est une pédagogie de la parole qui renvoie les représentations à la praxis langagière concrète à l'œuvre dans la prise de conscience individuelle et collective. Cet acte fondamental d'autodétermination seul peut s'effectuer dans la langue qui émerge de l'effort du sujet pour devenir principe créatif, pour être commencement originaire du sens, c'est-à-dire, seul peut se faire en langue native.

\section{Conclusion}

Pour construire un projet pédagogique de libération, Fiche couple sa théorie de l'apprentissage à la destinée sociale d'une communauté historiquement située. À cet égard, la rationalité éducative de l'Aufklärung avait éprouvé une limitation : si elle s'était vouée à découvrir les structures transcendantales

20 Face à l'Urvolk, le Ausland ne se définit pas par sa provenance depuis l'extérieur des limites empiriques de la nation (qu'il s'agisse de déterminations territoriales, biologiques ou d'ascendance historique) et une imposition étrangère peut parfaitement venir « du dedans » de la communauté nationale. Comme l'affirme Jean-Christophe Goddard: « [...] l'envahisseur, l'Ausland, parce qu'il vient de l'extérieur du Land pour le conquérir, est aussi la Nichtursprunglichkeit, la négation du principe aborigène : il est par essence non-natif, non pas au sens où il viendrait d'ailleurs, aurait une autre origine géographique, mais parce qu'il est ontologiquement étranger à toute genèse, toute médiation - il est, pour utiliser un lexème fichtéen, sans " Durch ». L'envahisseur vient toujours de nulle part, annule par le fait brutal de son acte d'invasion toute provenance, toute primitivité ou nativité - il n'a d'existence que factuelle. » (Goddard, 2012, p. 2). 
de l'éducabilité humaine, elle manquait - et en cela Kant en est peut-être l'expression la plus significative ${ }^{21}$ - d'une réflexion sur les conditions génétiques d'un perfectionnement autonome des sociétés. Mais il ne s'agit point de substituer à une éducation de principes universels, une instruction contextuelle de reproduction des coutumes et traditions, qui trouverait dans la nation le dépositaire d'une vertu substantielle qu'il est nécessaire de restaurer ${ }^{22}$. Bien autrement, il est question de penser la liberté humaine et son autoformation dans l'inexorable caractère situationnel de sa manifestation, de réactiver la puissance de la vie originaire dans et par son affectation à une atmosphère sociale concrète. En ce sens, il n'y a pas de priorité ontologique de la nation, et le romantisme qui promeut l'exaltation du contenu empirique de la collectivité ne fait que bloquer l'esprit dans la reproduction d'une silhouette immobile de soi-même, dans la réitération caricaturale d'une identité fantasmée.

En ce sens, bien que Fichte présente son projet comme un programme d'éducation nationale, il le définit surtout par son caractère populaire, par son rapport constitutif à la vie présente du peuple (GA, I, 10, p. 111). Un tel horizon éducatif n'est pas à comprendre comme une assimilation de grandes masses à un cadre civilisationnel préétabli, mais comme un travail de repère et activation des possibilités créatives des sujets pour la transformation de soi et de sa réalité sociale. Une pédagogie de la culture - et une école conséquente avec celle-là - divorcée des capacités autonomes des individus et des groupes sociaux ne peut avoir d'autre résultat que l'imposition hétéronome d'un système de principes abstraits qui vient étayer la maximisation technique

21 II serait sans doute injuste d'attribuer à la pédagogie kantienne l'impérialisme culturel des projets de domination européenne de l'époque, et Fichte - à la différence, peut-être, d'Hegel - ne pointe jamais la philosophie de son maître comme responsable de la dérive despotique de la pensée des Lumières. Fichte est conscient de l'effort de Kant pour développer une proposition pédagogique qui rend compte de la médiation entre la volonté morale et les volontés empiriques; c'est là où réside la réflexion anthropologique kantienne sur l'histoire en tant qu'éducation du genre humain qui habilite non pas la réalisation de la destinée morale, mais la possibilité de celle-ci dans une société civile juridique et culturellement éclairée. Kant ne s'est donc pas borné à un dualisme insurmontable entre nature et liberté, comme certains ont cru le voir. Cependant, s'il a thématisé la transition [Übergang] d'un domaine à l'autre (Cf. Kant, Critique du Jugement, AA, V, pp. 175-176) il s'est limité aux conditions transcendantales d'un tel passage. Dans une déduction qui va du devoir-être de la loi à la possibilité indéterminée de l'agir moral matériel - c'est-à-dire, parce que je dois alors je peux (Cf. Ibid, p. 176 ou encore Kant, La religion dans les limites de la simple raison, AA, VI, p. 50) - le texte kantien se prive d'une réflexion philosophique sur la genèse situationnelle de ce pouvoir-être dans la liberté incarnée des acteurs historiques. Ainsi, d'après Fichte, il faut dépasser la lettre kantienne à cet égard pour fournir un savoir des conditions concrètes, pragmatiques, contextuelles de la moralisation des subjectivités pratiques effectives.

22 Friedrich Schlegel est peut-être la référence la plus claire de l'idée romantique d'après laquelle le travail de I'histoire est celui de récupérer un Âge d'or perdu, présentée sous le signe d'une communauté immédiate entre Dieu et les êtres humains: "L'espoir religieux en une véritable et complète restauration [Wiederherstellung] de l'Âge historique d'un État chrétien et une science chrétienne constitue la conclusion de cette philosophie de l'histoire. » (Schlegel, 1988, p. 419). 
du régime égoïste de la propriété. La construction des espaces de sociabilité prétendument universels et neutres qui demeurent indéterminés à l'égard de l'adhésion des sujets empiriques - ce qui explique l'insuffisance kantienne de la Öffentilichkeit comme milieu pour le perfectionnement réciproque des individus - peut finalement se traduire, dans les faits, par une neutralisation de tout ce qui résiste la gestion technocratique du commun. Remarquable description de Fichte, lorsqu'il définit les universalismes despotiques comme ceux qui « ne parviennent pas à saisir quoi que ce soit dans l'existence présente ; ce qui existe, ils veulent seulement le détruire, et engendrer partout autour d'eux un vide où ils ne peuvent jamais que reproduire leur propre forme [...] » (Idem, p. 276).

Il est important de souligner le fait que Fichte ne va jamais renoncer au principe de la pédagogie des Lumières qui se fonde dans l'universalité de la destination spirituelle. La Bestimmung humaine demeure la même dans toutes les tentatives, des individus et des collectifs, pour se perfectionner mutuellement dans la construction d'un monde libre. La philosophie peut même tracer - comme le fait Fichte dans les Grundzüge - un plan universel [Weltplan] (GA, I, 8 p. 197), une épocalité du développement de la raison, non pas pour pointer une communauté de destin déterminée, mais pour signaler un temps présent comme point milieu [Mittelpunkt] à l'égard duquel il est possible de juger les efforts et les résistances de la construction d'une réalité mondaine à l'image de la raison (GA, I, 8 p. 206). Mais tout se joue dans la pragmatique de l'appréhension figurative de cet horizon spirituel, que Fichte met en œuvre dans le projet pédagogique des Discours. En termes clairs: l'universalité spirituelle telle que Fichte la conçoit ne peut se manifester que dans une pluralité de constructions civilisationnelles, en tant que reflets, ou « miroitements $»^{23}$, de la vie une de l'humanité. L'homogénéisation civilisatrice, qui surimpose une image (sociale, politique, esthétique) comme figuration définitive de la raison, inhibe l'autodiversification infinie de l'esprit, anéantit les «spécificités invisibles » des peuples, détruit ce « qui les relie à la source de la vie originaire »(GA, I, 10, p. 273).

23 Pour reprendre une expression de la explication précise de J-C. Goddard : " II est très remarquable que le nationalisme des Discours à la nation allemande est d'autant plus affirmé et justifié qu'il repose sur cette conception d'une révélation de l'Absolu dans ce miroitement, ce scintillement infini de la diversité des réalités individuelles, en lequel chacune, comme un éclat d'Infini, reçoit une valeur sacrée, qu'elle ne peut refuser aux autres, n'étant l'image de Dieu qu'à condition d'être rapportée à ce principe de la libre production d'images à partir de la lumière de l'Esprit par laquelle à la fois s'esquisse toute image déterminée et se dissout la prétention de chacune d'entre elles à une existence séparée, à être l'unique image de Dieu. » (Goddard, 1999, p. 214). 
Fichte nous offre ainsi une réflexion pédagogique attentive aux relations interculturelles et aux différentiels de pouvoir qui les parcourent. Si l'éducation $\mathrm{du}$ genre humain, comme pratique d'apprentissage, mais aussi comme paradigme du développement historico-culturel, ne s'enracine pas dans les processus effectifs d'autodétermination, elle sera un instrument aliénant au service des pouvoirs factuels. Cela n'aboutit aucunement au fait qu'un perfectionnement réciproque entre des communautés culturellement diverses soit impensable ${ }^{24}$. Toutefois, il ne faudrait pas chercher le fondement d'une telle éducation interculturelle dans la seule intellection d'un eidétique transversale, mais dans les créations autonomes, individuelles et collectives ${ }^{25}$, qui préfigurent, dans leurs pratiques situées, des modèles susceptibles de rapprocher des humanités différentes ${ }^{26}$. Au lieu d'une éducation universelle pour la liberté, dont le formalisme peut être instrumentalisé pour l'expansion empirique de l'égoïsme, Fichte nous propose une pédagogie de la libération, sous laquelle la destination de l'humanité ne se manifeste pas dans la systématisation de sa pureté rationnelle, mais dans son événementialité historique, immanente à l'autodétermination des peuples.

\section{Bibliographie}

ANDLER, Ch. « Le Pangermanisme philosophique ». Paris: Conard, 1917. ASMUTH, C. « Bild des Bildes des Bildes: Fichtes radikal konstruktivistische Bildtheorie». In: NEUBER, S., VERESSOV, R. (orgs.). Das Bild als Denkfigur. Funktionen des Bildbegriffs in Geschichte der Philosophie. München: Wilhelm Fink, 2010, pp. 153-167.

24 Comme des exemples positifs d'un échange interculturel on a déjà mentionné l'échange linguistique et culturel qui permet aux germains d'adopter des principes et des institutions issus du monde romain (GA. I, 10, pp. 206-207). On peut encore mentionner le rapport éducatif autonome que la nation allemande doit entretenir vis-à-vis de l'Antiquité classique, pour autant que cet héritage soit vivifié par une nouvelle créativité autochtone (GA. I, 10, p. 167).

25 C'est dans un tel sens que Marc Maesschalck propose de lire, chez le Fichte de cette période, l'achèvement de l'eidétique transcendantale dans une réflexion sur les postulats de la pratique sociale des libertés humaines: " C'est par la réalisation d'un pouvoir d'autodétermination visant un ordre collectif que pourra se juger l'idée d'une unité de destin de l'humanité, grâce à l'échange des cultures qui pourra résulter de la vie de ces entités autonomes. » (Maesschalck, 1996, p. 287)

26 L'objectif du premier des Dialogues patriotiques est justement celui d'exposer l'implication réciproque du patriotisme et du cosmopolitisme, compris correctement: « Elle [la volonté de la destination humaine] ne peut toutefois intervenir que dans le milieu qui l'entoure immédiatement, c'est-à-dire, là où elle existe et vit comme force vivante ; [...] Et de cette façon, tout esprit cosmopolite devient tout à fait nécessairement, par cette limitation à la Nation, patriote ; et tout individu qui dans sa nation serait le patriote le plus puissant et le plus actif est précisément, par là même, le citoyen du monde le plus actif ». (Dialogues Patriotiques, GA. II, 9 , p. 400). 
BASCH, V. « La philosophie et la littérature classiques de l'Allemagne et les doctrines pangermanistes ». Revue de Métaphysique et de Morale, Nr. 22, 1914, pp. 711-793.

BERGMANN, E. « Fichte und der Nationalsozialismus ». Breslau: Hirt, 1933.

BERLIN, I. "The Crooked Timber of Humanity". London: Murray, 1992.

CUNEEN, N. «L'actualité de la politique fichtéenne de l'éducation ». Interpretationes, Vol. VI, Nr. 1-2, 2016, pp. 152-176. https://www.cupress.cuni.cz/ink2_stat/dload. jsp?prezMat=103127.

DUMONT, L. « Essais sur l'individualisme ». Paris: Seuil, 1992.

FICHTE, J-G. « Gesamtausgabe der Bayerischen Akademie der Wissenschaften». Ed. Fuchs, H. Gliwitzky, R. Lauth, P. K. Schneider. Stuttgart-Bad Cannstatt: FrommannHolzboog, 1962ff.

FUCHS, E. « Fichte: Stammvater des deutschen Nationalismus? ». Fichte Studien, Nr. 35, 2010, pp. 267-284.

GARCIA, L-F. « La philosophie comme Wissenschaftslehre : le projet fichtéen d'une nouvelle pratique du savoir ». Hildesheim: Olms, 2018.

GODDARD, J-C. «Fichte o la revolución aborigen permanente». Revista de Estud(i) os sobre Fichte, Nr. 4, 2012. https://journals.openedition.org/ref/294?lang=pt.

GODDARD, J-C. «La résistance au pouvoir dans la pensée de Fichte ». In: Le Pouvoir. Ed. J-C. Goddard et Mabille, B. Paris: Vrin, 1994, pp. 163-177.

GODDARD, J-C. « La philosophie fichtéenne de la vie ». Paris : Vrin, 1999.

GUEROULT, M. «Etudes sur Fichte». Paris : Aubier-Montaigne, 1974.

HEIMSOETH, H. « Fichte ». München: Reinhart Verlag, 1923.

KANT, I. « Kritik der reinen Vernunft », Werke in Zehn Bände, Band 3. Darmstadt: Wissenschaftliche Buchgesellschaft, Darmstadt, 1968.

KANT, I. «Gesammelte Schriften». Ed. Bd. 1-22 Preussische Akademie der Wissenschaften, Bd. 23 Deutsche Akademie der Wissenschaften zu Berlin, ab Bd. 24 Akademie der Wissenschaften zu Göttingen, Bd. 25 Berlin-Brandenburglichen Akademie der Wissenschaften, Berlin, 1902ff. [AA dans le texte].

LANDENNE, Q. «Classes sociales, élites savantes et émancipation populaire chez Fichte ». Tumultes, Nr. 40, 2013, pp. 241-257. https://www.cairn.info/revue-tumultes2013-1-page-241.htm.

LAUTH, R. «Der letze Grund von Fichtes Reden an die deutsche Nation». Fichte Studien, 4, 1992, pp. 197-230.

LEBRUN, G. « Kant et la fin de la métaphysique ». Paris: Armand Colin, 1970.

LOSURDO, D. « Hegel et la catastrophe allemande ». Paris: Albin Michel, 1994.

MAESSCHALCK, M. « Droit et création sociale chez Fichte ». Louvain: Peeters, 1996. MAESSCHALCK, M. " Pour une éthique des convictions ». Bruxelles: Publications des Facultés universitaires Saint-Louis, 1994.

MAESSCHALCK, M. "Religion et identité culturelle chez Fichte ». Hildesheim: Olms, 2000.

PHILONENKO, A. « L'œuvre de Fichte ». Paris: Vrin, 1984.

POPPER, K. «The open society and its enemies ». London: Routledge and Kegan Paul, Tome II, 1945. 
RENAUT, A. «L'idée fichtéenne de nation ». In: Cahiers de Philosophie Politique et Juridique, Nr. 14, 1988, pp. 189-205.

SCHLEGEL, F. " Philosophie der Geschichte» in « Kritische Aufgabe». Paderborn: Ferdinand Schöningh Verlag, Bd. IX, 1988.

TALMON, J.-L. « Die Geschichte der totalitären Demokratie», Bd. 2, «Politischer messianismus, Die Romantische Phase», Köln: Opladen, 1963.

TORRES FILHO, R. «O espírito e a letra. A crítica da imaginação pura em Fichte». São Paulo: Ática, 1975.

TREITSCHKE, H. von. « Fichte und die nationale Idee». In: «Ausgewählte Schriften», Bd 1, Leipzig: Hirzel, 1907.

VERMEIL, E. «Un ancêtre de l'autarcie hitlérienne. J.G. Fichte ». L'Europe Nouvelle, Nr. 22, 1939. 
